\title{
Correspondence
}

\section{Wrist actigraphy in anesthesia}

To the Editor:

Intraoperative assessment of sedation/awakening is imprecise, and drug administration can, consequently, be either too little or too much. Changes in respiratory, hemodynamic and muscle tone do not appear instantaneously during induction and emergence from general anesthesia when their instant recognition is vital, especially after mishaps. End-tidal $\mathrm{CO}_{2}$ and pulse oxymetry are secondary events, the recordings of which appear 15-30 sec after they have changed. Direct arterial pressure monitoring follows cardiovascular alterations rapidly, but is rarely used in healthy patients. Continuous muscular status monitoring, especially during induction, intubation and awakening, would be invaluable but has not been utilized routinely in the anesthetic setting.

An electronic wrist actigraph (Figure 1, Ambulatory Monitoring Inc., Ardsley, NY, USA $)^{1}$ was compared to conventional clinical measurements and the anesthesiologist=s observations during anesthesia/sedation. It senses and records baseline tone patterns and later modifications, including even the slightest limb movements, and enables data storage. ${ }^{2}$

Actigrams were correlated to corresponding clinical vital signs (Figure 2A-D). Actigraphy-demonstrated on- ward activity was more than four times higher than after premedication. Shortly after midazolam (3$6 \mathrm{mg}$ ) or propofol $(25-75 \mathrm{mg})$ iv administration, limb movement dropped to baseline levels or below. These changes appeared more than three minutes before non-invasive blood pressure decreased and more than one minute before decreases in heart and respiratory rates. The anesthesiologist identified pain more than two to four minutes and awakening more than one minute after they had been detected actigraphically.

On-line actigraphy appears to supplement conventional monitoring with more rapid sedation/awakening information potentially bestowing greater patient safety.

Avi A. Weinbroom MD

Ron Ben-Abraham MD

Jacob Zomer BSC

Tel Aviv, Israel

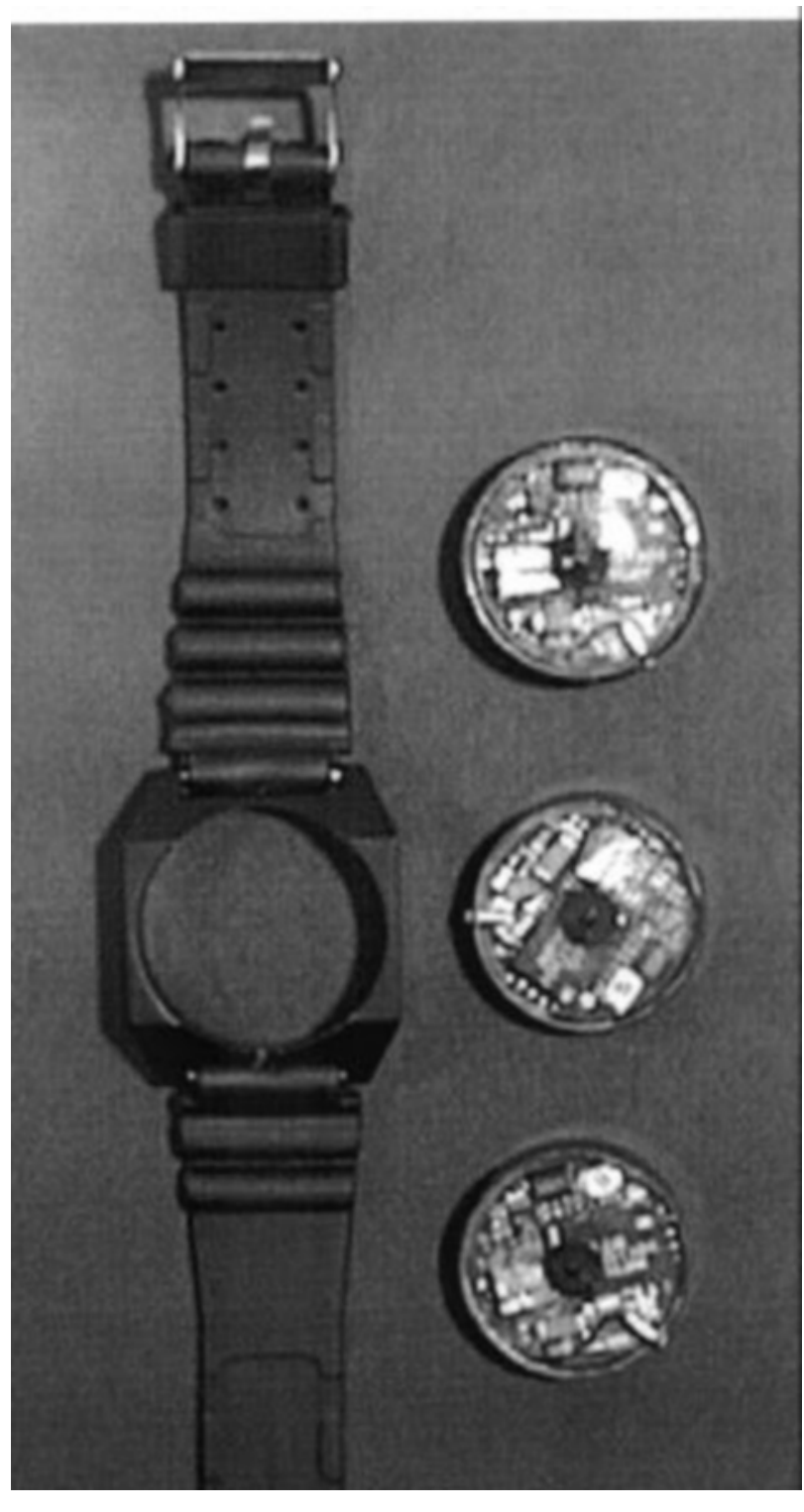

FIGURE 1 Wrist actigraph.

\section{References}

1 Sadeh A, Lavie P, Scher A, Tirosh E, Epstein R Actigraphic home-monitoring sleep-disturbed and control infants and young children: a new method for pediatric assessment of sleep-wake patterns. Pediatrics 1991; 87: 494-9. 

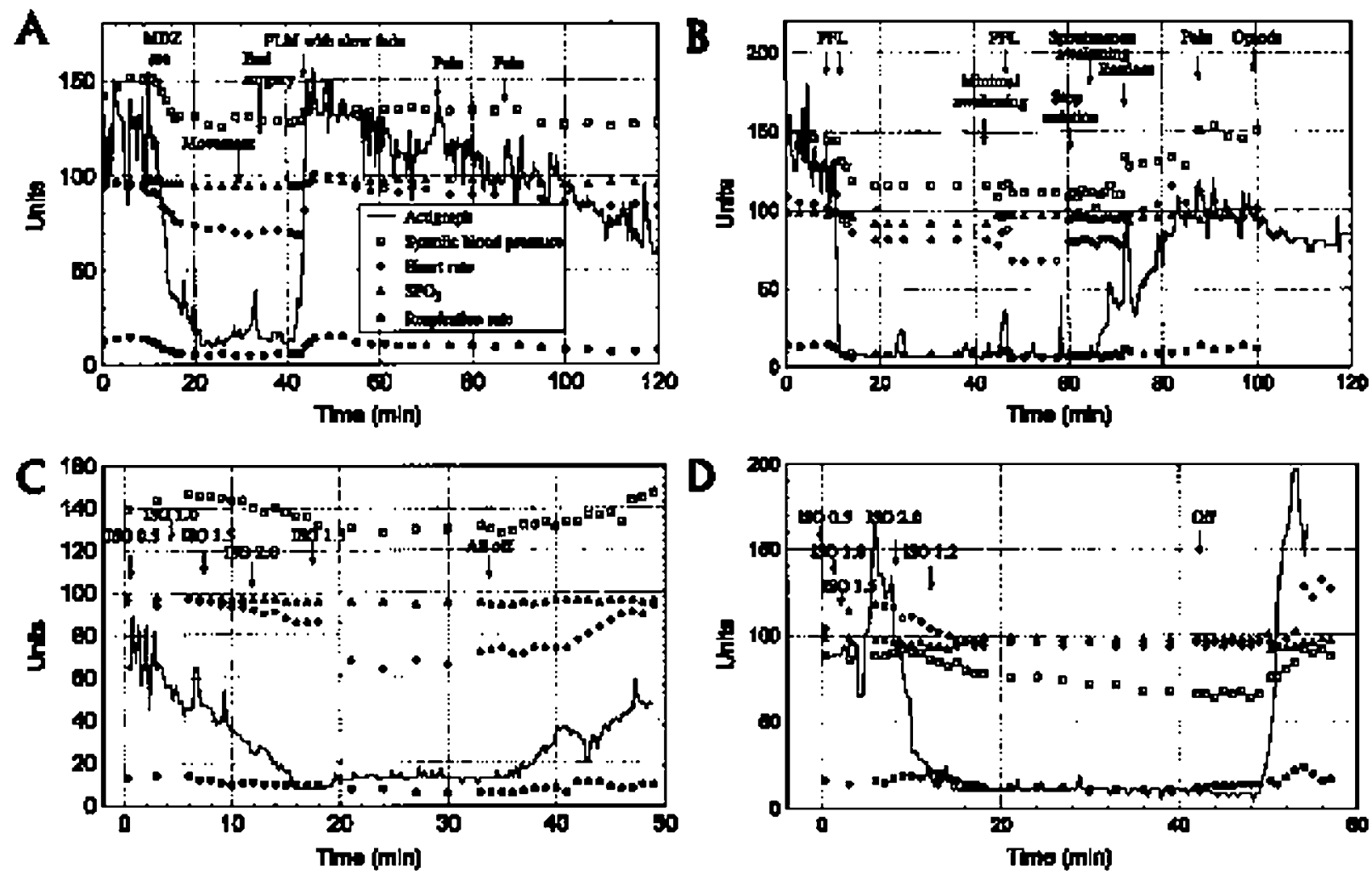

FIGURE 2 Actigrams and vital signs of patients undergoing surgery under monitored (epidural/spinal) sedation (A, B) and general anesthesia $(\mathrm{C}, \mathrm{D})$. Movement occurred during surgery in patient A. Note that the visible changes in the actigrams preceded equivalent changes in all other vital signs in all patients.

$\mathrm{MDZ}=$ midazolam; PFL=propofol; ISO=isoflurane; FLM=flumazenil; units: activity=arbitrary units; blood pressure=mmHg; heart rate=beats@min ${ }^{\mathrm{B}} ; \mathrm{SpO}_{2}=\%$; respiration rate=breaths@ $\min ^{\mathrm{B}}$.

2 Middlekoop HAM. Actigraphic Assessment of Sleep and Sleep Disorders. Delft: Eburon Publishers, 1994.

\section{Syringe contamination by propofol: a possible mechanism}

To the Editor:

Propofol, though a most popular induction agent providing an early awakening of anesthetized patients, ${ }^{1}$ has been shown to promote the growth of bacteria ${ }^{2}$ and fungi ${ }^{3}$ which might result in postoperative infection and sepsis. Breaking of ampoules, without taking aseptic measures, is the most common mode of extrinsic contamination. ${ }^{4}$ We report another possible source of such a contamination, which often goes unnoticed.

While administering propofol through an $i v$ cannula, a residual amount of medication stays in the drug port. The residual propofol, measured randomly in 50 patients, ranged between $0.05-0.15 \mathrm{~mL}$. When a subsequent drug is injected through the same port, the residual propofol migrates up into the syringe. Eventually, during prolonged procedures, the migrated propofol may lead to extrinsic contamination of subsequent drugs. This phenomenon is better noticed initially when propofol travels up in form of a thin streak followed by a "mushrooming effect" a la nuclear fission (Figure). Such contamination may contribute to postoperative infection and sepsis.

Manufacturers recommend that propofol be used within six hours of its handling, in a single patient, and not be stored thereafter. Sosis and Braverman ${ }^{3}$ demonstrated that the number of colony forming units of staphylococcus aureus were significantly greater when inoculated in sterile $0.9 \%$ saline when compared to solutions containing other anesthetics 